\title{
No Impact of the Increased Number of Cardiovascular Risk Factors on Thrombosis and Survival in Polycythemia Vera
}

\author{
Ivan Krečak ${ }^{a}$ Martina Morić Perić ${ }^{b}$ Ivan Zekanović ${ }^{b}$ Hrvoje Holik ${ }^{c}$ \\ Božena Coha ${ }^{c}$ Velka Gverić-Krečak ${ }^{a}$ Marko Lucijanićd \\ ${ }^{a}$ Department of Internal Medicine, General Hospital of Šibenik-Knin County, Šibenik, Croatia; \\ ${ }^{\mathrm{b}}$ Department of Internal Medicine, General Hospital of Zadar, Zadar, Croatia; ' Dr. Josip Benčević \\ General Hospital, Slavonski Brod, Croatia; dUniversity Hospital Dubrava, Zagreb, Croatia
}

\section{Dear Editor,}

We have read the article by Mancuso et al. [1] evaluating the impact of cardiovascular (CV) risk factors on overall survival (OS) in polycythemia vera (PV) patients with great interest. The authors have presented univariate survival analysis on a cohort of $165 \mathrm{PV}$ patients demonstrating inferior OS in PV patients with 1 or more $\mathrm{CV}$ risk factors. Interestingly, even though $\mathrm{CV}$ risk factors were recognized as risk factors for thrombosis in PV, with different CV factors differently affecting the type of thrombosis (arterial vs. venous) [2-4], their presence was not included in the European Leukemia Net (ELN) risk assessment [5]. To make things more complicated, hydroxyurea and phlebotomies seem to demonstrate different preventive effects on the type of thrombosis, with hydroxyurea being more effective in high-risk patients and more potent in the arterial district $[6,7]$. Furthermore, despite the increasing pool of evidence that interferons may exert disease-modifying abilities, whether to treat low-risk PV patients with cytoreduction still remains a matter of debate [8]. With this in mind, the study by Mancuso et al. [1] is surely relevant and timely, as it aimed to define whether the increasing number of $\mathrm{CV}$ risk factors in PV may be associated with a higher frequency of thrombosis and an inferior OS. Here, we would like to discuss the results of this study and to present our data which may suggest different conclusions.

Previous studies have recognized that advanced age, leukocytosis, and prior thrombosis were the most important risk factors to negatively affect both OS and throm- bosis in PV [9]. On the other hand, the association of the increasing number of $\mathrm{CV}$ risk factors with decreased $\mathrm{OS}$ seems plausible, as both arterial hypertension and diabetes have been shown to reduce life expectancy in the general population $[10,11]$ and $P V$ patients may be no exception. In addition, it seems that the authors' conclusion that the increasing number of $\mathrm{CV}$ risk factors may be associated with an increased risk of thrombosis in PV is based on a feeling, rather than on direct statistical evidence, as the authors presented pooled frequency of both baseline (49 in total) and postdiagnosis thrombotic incidents (only 16 in total) without formal statistical tests that would support the conclusions [1]. In our opinion, the paper would benefit from a presentation of Kaplan-Meier time to thrombosis (TTT) curves stratified by the number of $\mathrm{CV}$ risk factors as was shown for OS. It should also be noted that the authors did not provide multivariate analyses to demonstrate that the increasing number of $\mathrm{CV}$ risk factors independently affects survival and thrombosis in $\mathrm{PV}$ when compared to already established risk factors (age, prior thrombosis, leukocytosis), as confounding and patient selection bias may play substantial roles and affect conclusions in retrospective datasets.

To address the same issues, we have retrospectively included JAK2-positive PV patients diagnosed according to 2016 World Health Organization criteria [12] from 3 community hospitals in Croatia. Patients were included in the period between May 2000 and January 2019. Demographic and clinical data were recorded at the time of diagnosis. CV risk factors were defined as presence of ar- 


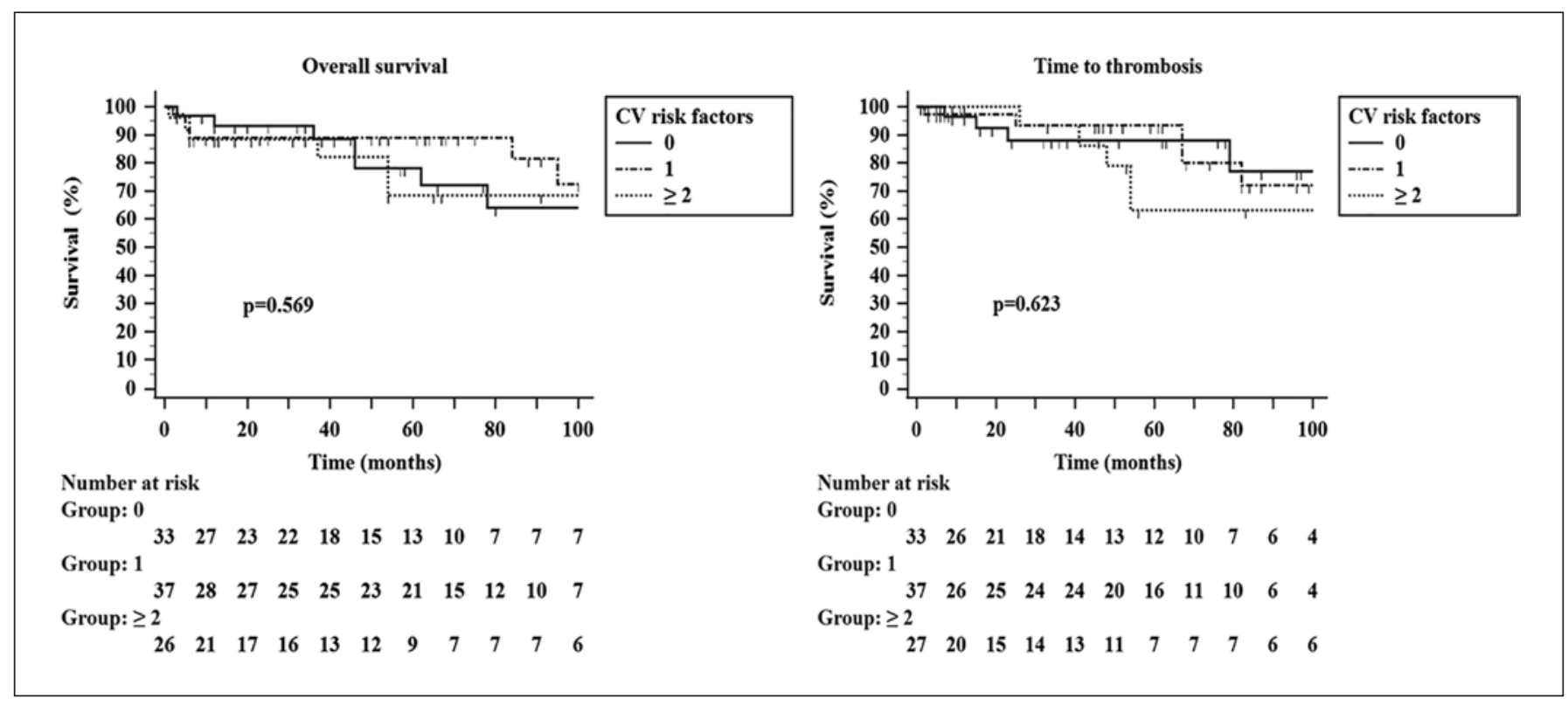

Fig. 1. The impact of the increasing number of cardiovascular $(\mathrm{CV})$ risk factors on overall survival and time to thrombosis in polycythemia vera patients.

terial hypertension, diabetes, hyperlipidemia, or smoking. High-risk disease was defined as age $>60$ years of age and/or history of thrombosis [5]. All patients were treated with phlebotomies to maintain the hematocrit $<45 \%$ and with low-dose aspirin, as per ELN guidelines [5]. Categorical variables were compared with the $\chi^{2}$ test, whereas the Kruskal-Wallis test was used to compare continuous variables among different patient categories. Survival analyses were screened with custom-made MS Excel workbook [13] and performed using the methods of Kaplan and Meier and the Cox regression analysis. TTT was calculated as the time from diagnosis until thrombosis (arterial or venous) or last follow-up (with patients who died being censored at the time of death), whereas OS was determined from the time of diagnosis until death or last follow-up visit. Arterial thromboses were defined as myocardial infarction, transitory cerebral ischemic attack, acute cerebral ischemic stroke, or acute peripheral arterial occlusion, whereas venous thromboses were defined as peripheral deep vein thrombosis and/or pulmonary embolism. Statistical calculations were performed with MedCalc Statistical Software ${ }^{\mathrm{TM}}$ (version 18.11.6, Ostend, Belgium). Significant $p$ value was set at $<0.050$.

A total of 97 JAK2-positive patients were included; the median age was 68 years (range 21-92) with 46 (47.4\%) females. Twenty-six $(28.6 \%)$ patients had a history of thrombosis, 48 (49.5\%) were treated with hydroxyurea, 76 (78.4\%) had high-risk disease, and 65 (67\%) had CV risk factors. The median follow-up was 54 months (range 1-231) and 59.5 months (range 2-231) for the entire cohort and for the surviving patients, respectively. Twenty (20.6\%) patients died and $16(16.5 \%)$ patients experi- enced thrombosis during the follow-up. A total of 33 (34\%), 37 (38\%), and $27(27.8 \%)$ patients had 0,1 , or 2 or more $\mathrm{CV}$ risk factors, respectively. The most frequent $\mathrm{CV}$ risk factor was arterial hypertension, found in 61 patients (83.6\%), followed by hyperlipidemia in 21 (32.3\%), smoking in $9(13.8 \%)$, and diabetes in 8 patients $(12.3 \%)$. There was no difference according to age $(p=0.396)$ or sex $(p=$ 0.565 ) among patients with a different number of $C V$ risk factors. Patients with CV risk factors more often had a history of thrombosis ( 80 vs. $63.9 \%, p=0.048$ ); however, the increasing number of $\mathrm{CV}$ risk factors did not correlate with a higher frequency of prior thrombosis $(p=0.409)$. Also, the number of CV risk factors did not correlate with blood cell counts at diagnosis ( $p>0.050$ for all analyses).

In survival analyses, both prior thrombosis (HR 4.3; $p=0.037)$ and age $>70$ years (HR 14.78; $p<0.001)$ were associated with an inferior OS. Similarly, age $>70$ years (HR 6.54; $p=0.010$ ) and high-risk disease (HR 6.81; $p=$ $0.009)$ were associated with a shorter TTT. On the other hand, the increasing number of CV risk factors was not associated with an inferior OS $(p=0.569)$ nor with shorter TTT ( $p=0.623)$, as shown in Figure 1. These results were also confirmed in high-risk patients ( $p=0.968$ for OS and $p=0.949$ for TTT) and in low-risk patients for OS ( $p=0.313)$, whereas none of our low-risk PV patients experienced thrombosis during the follow-up. Finally, we analyzed whether any of the individual CV risk factors may be particularly associated with an inferior OS or shorter TTT but found no significant associations $(p>$ 0.050 for all analyses). Thus, we were not able to replicate the results of Mancuso et al. [1]. The same limitations of a retrospective design and small number of patients and 
events and possible biases as mentioned previously can be present in our study as well. Data emerging from other independent cohorts of patients are eagerly awaited to provide further insight into this important issue.

We would like to congratulate the authors on their effort and the impetus on future studies evaluating the impact of the rising number of $\mathrm{CV}$ risk factors on thrombosis and OS in PV. If possible, we would like to suggest to the authors to further analyze whether the impact of CV risk factors (and their increasing number) remains independently (of advanced age, history of thrombosis, and leukocytosis) associated with both inferior OS and shorter TTT in the multivariate Cox regression models.

\section{Statement of Ethics}

The study was performed in accordance with the Declaration of Helsinki and was approved by the Ethics Committees from all participating centers; General Hospital of Šibenik-Knin County (reference No. 01-3618/1-20), General Hospital Zadar (reference
No. 02-2025/20-6/20), and "Dr. Josip Benčević" General Hospital (reference No. 04000000/20-37). Due to the retrospective design of the study, informed consent was not obtained.

\section{Conflict of Interest Statement}

The authors have no conflicts of interest to declare.

\section{Funding Sources}

The authors received no funding.

\section{Author Contributions}

Ivan Krečak and Marko Lucijanić conceptualized the manuscript, treated the patients, collected data, performed statistical analyses, and wrote the first draft of the manuscript. Martina Morić Perić, Ivan Zekanović, Hrvoje Holik, Božena Coha, and Velka GverićKrečak treated the patients, collected data, and revised the manuscript. All authors approved the final version of the manuscript.

\section{References}

1 Mancuso S, Santoro M, Accurso V, Agliastro G, Raso S, Di Piazza F, et al. Cardiovascular Risk in Polycythemia Vera: Thrombotic Risk and Survival: Can Cytoreductive Therapy Be Useful in Patients with Low-Risk Polycythemia Vera with Cardiovascular Risk Factors? Oncol Res Treat. 2020;43(10):526-30.

2 Barbui T, Carobbio A, Rumi E, Finazzi G, Gisslinger H, Rodeghiero F, et al. In contemporary patients with polycythemia vera, rates of thrombosis and risk factors delineate a new clinical epidemiology. Blood. 2014 Nov; 124(19):3021-3.

3 Cerquozzi S, Barraco D, Lasho T, Finke C, Hanson CA, Ketterling RP, et al. Risk factors for arterial versus venous thrombosis in polycythemia vera: a single center experience in 587 patients. Blood Cancer J. 2017 Dec;7(12): 662.

4 Horvat I, Boban A, Zadro R, Antolic MR, Serventi-Seiwerth $R$, Roncevic $P$, et al. Influence of blood count, cardiovascular risks, inherited thrombophilia, and JAK2 V617F burden allele on type of thrombosis in patients with
Philadelphia chromosome negative myeloproliferative neoplasms. Clin Lymphoma Myeloma Leuk. 2019 Jan;19(1):53-63.

5 Barbui T, Tefferi A, Vannucchi AM, Passamonti F, Silver RT, Hoffman R, et al. Philadelphia chromosome-negative classical myeloproliferative neoplasms: revised management recommendations from European LeukemiaNet. Leukemia. 2018 May;32(5): 1057-69.

6 Barbui T, Vannucchi AM, Finazzi G, Finazzi MC, Masciulli A, Carobbio A, et al. A reappraisal of the benefit-risk profile of hydroxyurea in polycythemia vera: A propensitymatched study. Am J Hematol. 2017 Nov; 92(11):1131-6.

7 Barbui T, De Stefano V, Ghirardi A, Masciulli A, Finazzi G, Vannucchi AM. Different effect of hydroxyurea and phlebotomy on prevention of arterial and venous thrombosis in Polycythemia Vera. Blood Cancer J. 2018 Nov;8(12):124.

8 Kiladjian JJ, Barbui T. From leeches to interferon: should cytoreduction be prescribed for all patients with polycythemia vera? Leukemia. 2020 Nov;34(11):2837-9.

9 Tefferi A, Barbui T. Polycythemia vera and essential thrombocythemia: 2019 update on diagnosis, risk-stratification and management. Am J Hematol. 2019 Jan;94(1):133-43.

10 Preston SH, Choi D, Elo IT, Stokes A. Effect of Diabetes on Life Expectancy in the United States by Race and Ethnicity. Biodemogr Soc Biol. 2018;64(2):139-51.

11 Franco OH, Peeters A, Bonneux L, de Laet C. Blood pressure in adulthood and life expectancy with cardiovascular disease in men and women: life course analysis. Hypertension. 2005 Aug;46(2):280-6.

12 Arber DA, Orazi A, Hasserjian R, Thiele J, Borowitz MJ, Le Beau MM, et al. The 2016 revision to the World Health Organization classification of myeloid neoplasms and acute leukemia. Blood. 2016 May;127(20):2391405.

13 Lucijanić M. Survival analysis in clinical practice: analyze your own data using an Excel workbook. Croat Med J. 2016 Feb;57(1):77-9. 